\title{
Vision: Bias in Systematic Grammar-Based Test Suite Construction Algorithms
}

\author{
Christoff Rossouw \\ Division of Computer Science \\ Stellenbosch University \\ Stellenbosch, South Africa \\ 20723806@sun.ac.za
}

\author{
Bernd Fischer \\ Division of Computer Science \\ Stellenbosch University \\ Stellenbosch, South Africa \\ bfischer@sun.ac.za
}

\begin{abstract}
The core of grammar-based test suite construction algorithms is a procedure to derive a set of specific phrases, which are then converted into sentences that can be fed into the system under test. This process includes several degrees of freedom and different implementations choose different but ultimately fixed solutions. We show that these fixed choices inherently bias the generated test suite.

We quantify these biases and evaluate the effect they have on coverage over the system under test for which the test suite is constructed. We show that the effect of these biases remains prevalent in large real world grammars and systems, even when the test suites grow very large.
\end{abstract}

CCS Concepts: • Software and its engineering $\rightarrow$ Parsers; Syntax; Software testing and debugging; • Theory of computation $\rightarrow$ Grammars and context-free languages.

Keywords: test case generation

ACM Reference Format:

Christoff Rossouw and Bernd Fischer. 2021. Vision: Bias in Systematic Grammar-Based Test Suite Construction Algorithms. In Proceedings of the 14th ACM SIGPLAN International Conference on Software Language Engineering (SLE '21), October 17-18, 2021, Chicago, IL, USA. ACM, New York, NY, USA, 7 pages. https://doi.org/10.1145/ 3486608.3486902

\section{Introduction}

In grammar-based testing, the system under test (SUT) is executed over a test suite that is constructed from a contextfree grammar (CFG), which describes the structure of the SUT's inputs. The test suites can be constructed by deriving

Permission to make digital or hard copies of all or part of this work for personal or classroom use is granted without fee provided that copies are not made or distributed for profit or commercial advantage and that copies bear this notice and the full citation on the first page. Copyrights for components of this work owned by others than the author(s) must be honored. Abstracting with credit is permitted. To copy otherwise, or republish, to post on servers or to redistribute to lists, requires prior specific permission and/or a fee. Request permissions from permissions@acm.org. SLE '21, October 17-18, 2021, Chicago, IL, USA

(C) 2021 Copyright held by the owner/author(s). Publication rights licensed to ACM.

ACM ISBN 978-1-4503-9111-5/21/10 . .\$15.00

https://doi.org/10.1145/3486608.3486902

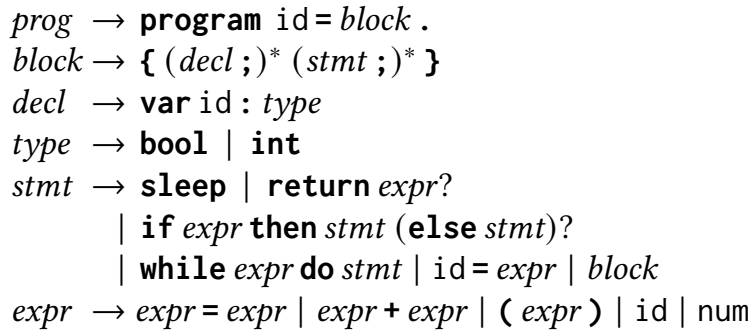

Figure 1. Example grammar $G_{\text {toy }}$ (adapted from [7])

sentences over the CFG in random or systematic fashion; we focus on the latter here.

The different systematic test suite construction algorithms are driven by different criteria, which obviously impact the results. For example, Purdom's algorithm [5, 6] constructs a minimal set of sentences such that each production is used at least once in a derivation, while the PLL coverage algorithm [11] constructs the set of shortest sentences with $S \Rightarrow^{*} \alpha A \omega \Rightarrow^{*} \alpha a \beta \omega \Rightarrow^{*} w$ for each non-terminal $A$ and each terminal $a \in \operatorname{first}(A)$.

These algorithms have several degrees of freedom and allow some implementation variants, which can impact their results in subtle and often unexpected ways. For example, Purdom's algorithm can use any rule $A \rightarrow \alpha$ still unused when $A$ is expanded. Likewise, the PLL coverage algorithm can use any derivation $A \Rightarrow^{*} a \alpha \Rightarrow^{*}$ av leading to a shortest yield $a v$ for $A$.

However, any implementation of a grammar-based test suite construction algorithm must ultimately resolve these choices in one way or another-typically deterministically, in some seemingly arbitrary but fixed manner (e.g., selecting symbols in alphabetic order or rules in textual order). Unfortunately, the resolution of these choices introduces biases into the generated test suites. Consider for example the grammar in Figure 1. This allows two different derivations of a shortest yield for stmt (stmt $\Rightarrow$ sleep and stmt $\Rightarrow$ return expr? $\Rightarrow$ return, respectively), and likewise for expr and type. Figure 2 shows on the left the test suite generated for this grammar by the generic cover algorithm shown in Algorithm 1, with rule coverage as criterion, textual rule order as strategy to choose between rules leading to different shortest yields, and minimal height derivation trees 
for embeddings. We can see that the selected shortest yield for stmt leads to a over-representation or bias in favor of sleep-statements and an underrepresentation or bias against return-statements: sleep occurs in eight of the fifteen generated tests, while return occurs only in the two tests that explicitly cover the corresponding rules.

These different implementation choices matter, since they can result in substantially different test suites for the same grammar and the same criterion, which can in turn result in substantially different coverage of the SUT. For example, for an SQL grammar with 586 rules the generic cover algorithm produces different test suites satisfying rule coverage that achieve between $22.1 \%$ and $26.2 \%$ statement coverage over the SQLite 3.36 .0 system, i.e., an $18 \%$ difference between worst and best case (see Table 2). Moreover, rule coverage leads in many cases to a better coverage than more comprehensive criteria such as context-dependent rule coverage (CDRC). The introduced biases therefore constitute a threat to validity for any experiments that compare different grammar-based testing algorithms based on coverage over a SUT, and make replication of experiments harder.

In this paper, we investigate such biases for a class of algorithms that systematically derive a set of target phrases $\Theta$ to satisfy different criteria over the derivations or the phrases, and embed each $\theta \in \Theta$ into a derivation $S \Rightarrow^{*} \alpha \theta \omega \Rightarrow^{*} w$ from the start symbol $S$ to a sentence $w$. We focus on a generic cover algorithm that has been proposed in several variations $[2,3,10]$ in the literature and that generalizes the PLL and CDRC coverage [4] algorithms.

Specifically, we focus here on two main kinds of bias that are related to the construction of the phrases and their conversion to sentences. The first kind of bias results from the way in which the target phrase is embedded and the nonterminals in the resulting sentential form are "grounded out", i.e., the construction of the derivation $S \Rightarrow^{*} \alpha \theta \omega \Rightarrow^{*} w$ for a given $\theta$. Test suite construction algorithms minimize these derivations wrt. some criterion because they aim at minimal tests. The choice of a minimization criteria, such as the length of the derivation or of the resulting word, or the height of the derivation tree, can have a large impact on the overall structure of the generated test suites (see Figure 2 for an example). The second kind of bias emerges when multiple minimal derivations (wrt. the chosen criterion) exist, and the algorithm arbitrarily picks one; this is the situation already described in the example above.

In order to measure the effect of these biases we consider the coverage achieved by a test suite over the SUT. We show that there is a large difference between test suites derived using shallowest derivations and those derived using shortest yield derivations. We also show that randomly choosing from a set of equivalent derivations leads to a large variance in coverage achieved. This leads us to caution that experimental results using coverage as a metric for comparing grammar-based test suite construction algorithms may easily be skewed by biases in the construction and that careful consideration of these biases is essential in performing fair comparisons.

However, on the flip side, we can also "play the odds" and exploit the variance of the algorithms. We show that we can significantly increase the coverage of the SUT if we merge the results of multiple runs of the algorithm under different choices into a combined test suite.

In this paper, we make four novel contributions. (i) We identify bias as a problem in systematic grammar-based test suite construction algorithms and show that it poses an hitherto unacknowledged threat to validity for experiments that compare different algorithms based on coverage over a SUT. (ii) We identify two different kinds bias, embedding bias and equivalent choice bias. (iii) We experimentally evaluate the effect they have on the coverage of the SUT for which the test suite is constructed, and show that the effects remain prevalent in a large real world grammar and system, even when the test suites grow very large. (iv) We show that we can exploit the variance by merging the results of multiple runs of the algorithm under different choices into a combined test suite that significantly increases the coverage of the SUT.

\section{Background}

Grammars. A grammar is a four-tuple $G=(N, T, P, S)$ with $N \cap T=\emptyset, P \subset N \times(N \cup T)^{*}$, and $S \in N$. We follow the notation of Aho et al. [1, Section 4.2.2], and denote non-terminals in $N$ by $A, B, C \ldots$, terminals in $T$ by $a, b, c \ldots$, grammar symbols in $V=N \cup T$ by $X, Y, Z$, words in $T^{*}$ by $u, v, \ldots$ and phrases in $V^{*}$ by $\alpha, \beta, \gamma, \ldots$

Derivations. We use $\alpha A \beta \Rightarrow_{G} \alpha \gamma \beta$ to denote that $\alpha A \beta$ derives $\alpha \gamma \beta$ by application of the rule $A \rightarrow \gamma \in P$, with $\Rightarrow^{k}$ denoting its $k$-fold repetition and $\Rightarrow^{*}$ its reflexive-transitive closure. A sentential form is a phrase $\alpha$ with $S \Rightarrow^{*} \alpha$, a sentence is a word $w$ with $S \Rightarrow^{*} w$. We also use a simultaneous derivation relation $\Rightarrow$, where $X_{1} \ldots X_{n} \Rightarrow \gamma_{1} \ldots \gamma_{n}$ if $X_{i} \rightarrow$ $\gamma_{i} \in P$ for all $X_{i} \in N$ and $\gamma_{i}=X_{i}$ for all $X_{i} \in T$. We denote its $k$-fold repetition $\Rightarrow^{k}$ by $b f s_{k}$ because it amounts to $k$ breadth-first rounds of rule applications [10].

The yield of a phrase $\alpha$ is the set of all words that can be derived from it, i.e., yield $(\alpha)=\left\{w \in T^{*} \mid \alpha \Rightarrow^{*} w\right\}$. The language $L(G)$ generated by a grammar $G$ is the yield of its start symbol, i.e., $L(G)=\left\{w \in T^{*} \mid S \Rightarrow^{*} w\right\}$. We generalize the usual definition of the first-set to first $(X)=\{Y \in V \mid$ $\left.X \Rightarrow^{*} Y \alpha\right\}$.

An embedding of $X$ in $A$ is a phrase $\alpha X \omega$ with $A \Rightarrow^{*}$ $\alpha X \omega \Rightarrow^{*} w$. It is called a shortest yield embedding if for all $A \Rightarrow^{*} \beta X \gamma \Rightarrow^{*} v$ we have $|w| \leq|v|$. It is called a shallowest embedding if $S \Rightarrow^{k} \alpha X \omega$, and there is no $i<k$ such that $S \Rightarrow^{i} \beta X \gamma$; hence, the length of a shallowest embedding corresponds to the height of a minimal derivation tree with 
applied rules. Note, however, that the maximal number of applications of each rule (which would minimize bias) is not compositional, and is therefore not compatible with the generic cover algorithm.

\section{Biases}

In statistics, a sample statistic (i.e., any quantity that is computed by aggregating values in a sample of an underlying population, such as the sample mean or variance) is biased if it is systematically different from the corresponding quantity of the entire population. Statistical bias has many forms, including modeling bias (e.g., using a normal distribution to model a population with long tails), observer bias (e.g., the Hawthorne-effect), or sampling bias (e.g., selecting participants with a landline phone number), although the latter is the most prevalent. It occurs (e.g., as self-selection, exclusion, or survivorship bias) whenever the sample is collected in a way such that specific groups of the underlying population are over-represented in the sample.

We focus on sampling bias here. More specifically, we consider as population the set of all test suites for a chosen coverage criterion and as sample a test suite constructed for a specific algorithm with specific fixed choices. The biased sample statistic that we are observing is the code coverage the generated test suite achieves over a SUT. We focus on the two types of fixed choices described below. Note that we do not consider any bias caused by different grammar variation and preprocessing steps (e.g., EBNF operator elimination) because these are under the full control of the user.

Embedding Bias. The generic cover algorithm and its variants use minimal derivations in the embedding (line 3) and completion (line 5) steps, and in some of the criteria. We compare the effects of shortest yield and shallowest derivations here. Shortest yield derivations $\Rightarrow_{\leq}^{*}$ induce a very strong bias towards $\epsilon$-productions, leaving optional elements of the grammar exercised much less often. Shallowest derivations $\Rightarrow{ }_{\sqsubset}^{*}$ induce a bias against deeply nested tests, leading to structurally simpler tests. This often corresponds to the shortest yield derivations, but not necessarily so; for example, in $G_{t o y}$ the shortest yield embedding of num is through a returnstatement, while the shallowest embeddings are through an if-, while-, or assignment statement. Note that the user can in principle deliberately choose the minimal embedding, taking these biases into account, although the choice is often hard-coded in the algorithm's implementation.

Regardless of the choice of embedding, it will lead to sampling bias since there will be an increased preference of sentences with the characteristics of the embedding in the test suite.

Equivalent Choice Bias. Regardless of the chosen minimal derivation method $\Rightarrow_{\leq}^{*}$, the algorithms need at some points to choose between a set of equivalent (wrt. $\Rightarrow_{\leq}^{*}$ ) options. If these choices are made systematically (e.g., based on textual rule order) rather than randomly, this introduces a classic sampling bias, and the generated test suite will be biased accordingly. If the choices are functionally determined by the grammar structure, the user could in principle control the bias by reformulating the grammar, but this becomes infeasible in practice.

\section{Experimental Setup}

In order to show the effect of the identified biases, we used an implementation of the generic cover algorithm to generate from two different target grammars (AMPL and SQLite) sets of equivalent but differently biased test suites for a series of different coverage criteria, and measured the code coverage over two corresponding code bases.

Design. For both targets, we converted the original EBNF grammars into plain BNF form, by introducing new nonterminal symbols for each occurrence of an EBNF operator, together with rules encoding the operators' definitions. From these BNF versions, we generated test suites satisfying the $p l l$, rule, cdrc, deriv [10], step $p_{3}$, and $b f_{2}$ coverage criteria; for AMPL, we also used $s_{e} p_{4}$, which grew too large for SQLite.

In order to evaluate the embedding bias, we consider the choice of minimal derivations as independent variable. For each of the 13 experiments, we varied this over $\Rightarrow_{\leq}^{*}$ and $\Rightarrow{ }_{\sqsubseteq}^{*}$.

In order to evaluate the equivalent choice bias, we consider the textual rule order as independent variable, since our implementation resolves ties between equivalent minimal yields and embeddings accordingly. More specifically, we created 100 variants of each grammar, by randomly shuffling the rule orders with different seeds; the number of unique test suites ranges from 72 to $100\left(\bar{x}_{\leq}=95.2, \sigma_{\leq}^{2}=121.3, \bar{x}_{\sqsubseteq}=\right.$ $\left.99.2, \sigma_{\sqsubseteq}^{2}=3.8\right)$. The average sizes of the different test suites are shown, in brackets next to the algorithm name, in Tables 1 and 2.

AMPL. AMPL is a small $(|N|=45,|T|=48,|P|=90)$ artificial programming language designed for a computer architecture course that was previously used in experiments by van Heerden et al. [10]. We obtained the 61 student compilers used in their experiments. These are small C programs comprising on average approx. 1300 LoC.

We ran each compiler individually over each of the respective test suites, executing each test individually, and used gcov to measure the cumulative line and branch coverage of each compiler over all tests in a test suite. We consider the averages over all 61 compilers as the dependent variables in the AMPL experiments.

SQLite. SQLite is a fully-featured SQL database engine implemented in C. We used the SQLite grammar (v3.8.x, $|N|=201,|T|=155,|P|=586)$ from the ANTLR grammar repository (https://github.com/antlr/grammars-v4) as target grammar and the C executable for SQLite (v3.36.0) compiled from sources (234229 LoC) as SUT. 
We ran the SUT over each of the respective test suites, executing each test as an individual SQL statement, and used gcov to measure the cumulative line and branch coverage over all tests in a test suite. We consider these values as the dependent variables in the SQLite experiments.

\section{Experimental Results}

Equivalent Choice Bias. Figure 3 and Tables 1 and 2 show a high variance in the code coverage under the different reordered grammar variants, especially for shallowest embeddings, and even though this decreases for larger test suites such as deriv, step $p_{3}$, or $b f_{2}$, it remains significant. For example, if we consider, for shallowest embeddings, the best line coverage achieved using rule coverage over SQLite (26.2\%), we see that this outperforms the average line coverage of all other criteria but step 3 (26.5\%)-which is almost two orders of magnitude bigger in terms of average test suite size.

Hence, any comparative study of such criteria and algorithms can be significantly skewed if the effect of bias is not taken properly into consideration; this is particularly relevant when different implementations are compared, where not all bias-inducing choices are necessarily known.

Figure 3 also shows that the coverage achieved is not generally normally distributed, with clusters often occurring near the minimum and maximum measured coverage. Test suites are thus more likely to achieve coverage near the extremes than near the average, reinforcing the bias effect.

Embedding Bias. Unlike for equivalent choice bias, the results for the different embeddings show noticeable coverage differences between AMPL and SQLite. For AMPL, we can see in Table 1 a large difference in the code coverage achieved between shallowest embeddings and shortest yield embeddings. The latter lead to a substantially higher average code coverage for the different criteria (0.9-2.3\%-points for line coverage, with $2.0 \%$-points on average, and 1.6-4.0\%-points for branch coverage, with $3.4 \%$-points on average) than the former, with a substantially smaller variance at the same time. However, the best variants under both embeddings achieve roughly the same coverage for all criteria.

For SQLite, the average code coverage achieved is approximately similar across all criteria (see Table 2), but Figure 3 shows that the coverage distributions for shallowest and shortest yield embeddings are very different for most criteria, and a two-tailed Mann-Whitney U test $(\rho<0.05)$ confirms that the difference in distributions is significant for each coverage criterion, with the exception of rule-coverage. Since shallowest embeddings have a large variance, several variants should be run to achieve reliable results, while shortest yield embeddings are more stable and should be preferred if the budget only allows a single run.

In our experiments none of the two embedding algorithm appears to universally outperform the other. Rather, the relative coverage achieved by either algorithm depends on the structure of the input grammar as well as the structure of the SUT. However, generally there are more possible shallowest embeddings than shortest yields, which is reflected in the greater observed coverage variance in test suites constructed using shallowest embeddings. These test suites thus often achieve better maximal coverage.

Randomization. The large variance in coverage is not necessarily only negative, and we can indeed exploit it to boost overall coverage by merging the various variants into one overall test suite, i.e. creating the union of the 100 different test suites. This obviously results in a larger test suite that achieves better coverage than the individual test suites. However, in certain cases it is even possible to create a combined test suite that has a smaller size and still achieves better coverage than some larger test suites for more complex criteria. For example, for SQLite the combined rule test suite for shallowest embedding, with a line coverage percentage of $26.5 \%$, achieves equivalent coverage to both $b f s_{2}$ and step $p_{3}$, which achieve an average line coverage of $26.1 \%$ and $26.5 \%$ respectively, while being only about one fifth and one third of their sizes, respectively.

\section{Conclusion}

We have shown that bias in grammar-based test suite constructions algorithms can have detrimental effects on the coverage achieved over an SUT. We have investigated the effects of two types of sampling bias present in grammar-based testing and shown that they remain prevalent, even in very large test suites and very large systems. We believe that these results are significant because context-free grammars are in practice used to test full systems with context-sensitive constraints, not just parsers We have also shown that, in some cases, it is possible to combine small biased test suites for a simple coverage criteria to achieve better coverage over the SUT than some larger, more complex test suites.

Future Work. The biases we have discussed here also appear in a similar way for automaton-based methods [8]; even random test suite generation methods also suffer from embedding bias, since they need to force termination. A different form of bias, structural bias, arises from rephrasing the grammar in a way that changes the structure, such as converting it to a different normal form [9]. We plan to investigate these biases, as well as biases occurring in further test suite generation algorithms [3, 5], including algorithms that algorithms that ensure that guarantee that the semantic constraints are satisfied (resp. violated) and so trigger more semantic actions.

We also plan to investigate further methods of exploiting bias to increase coverage, e.g., incrementally generating randomized test suites for a coverage criterion until coverage results stabilize, using different minimal derivations for the embedding and completion steps, or randomly sampling yields individually during completion. 


\section{Appendix: Detailed Results}
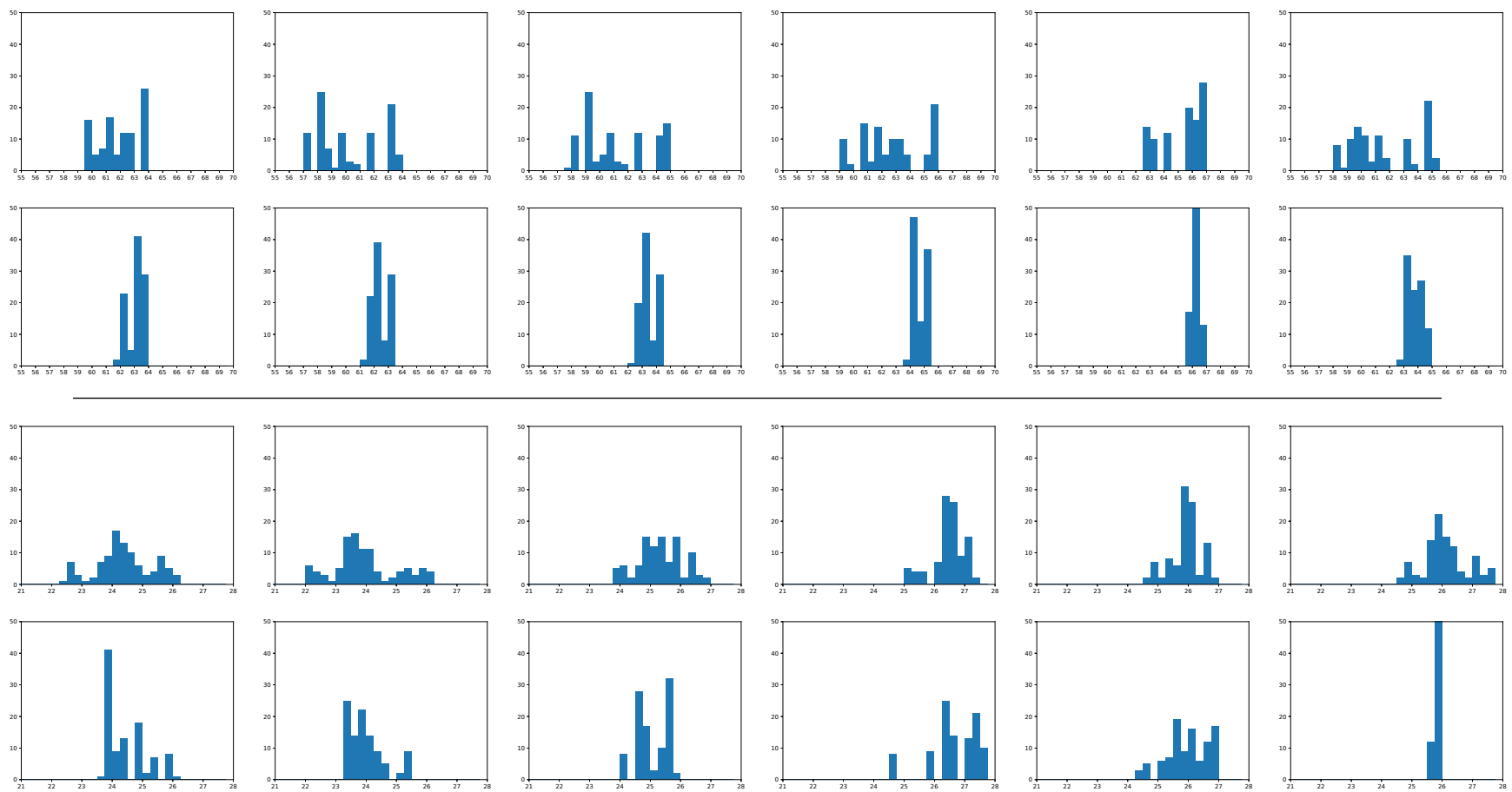

Figure 3. Statement coverage distribution for AMPL (above the line) and SQLite (below the line). For both AMPL and SQLite, shallowest embedding results are shown in the top row and shortest yield embedding results in the bottom row. Columns show, from left to right, results for pll, rule, $c d r c$, step $p_{3}$, deriv, and $b f s_{2}$ coverage. Each chart shows the observed statement coverage on the $\mathrm{x}$-axis and the corresponding number of test suites on the $\mathrm{y}$-axis.

\begin{tabular}{|c|c|c|c|c|c|c|c|c|c|c|c|c|c|c|}
\hline \multicolumn{15}{|c|}{ shallowest embedding } \\
\hline algorithm (tests) & \multicolumn{2}{|c|}{ pll (72.3) } & \multicolumn{2}{|c|}{ rule $(48.0)$} & \multicolumn{2}{|c|}{$\operatorname{cdrc}(81.0)$} & \multicolumn{2}{|c|}{$\operatorname{step}_{3}(182.0)$} & \multicolumn{2}{|c|}{ step $_{4}(429.0)$} & \multicolumn{2}{|c|}{ deriv (426.1) } & \multicolumn{2}{|c|}{$\mathrm{bfs}_{2}(150.0)$} \\
\hline coverage type & line & branch & line & branch & line & branch & line & branch & line & branch & line & branch & line & branch \\
\hline $\max (\%)$ & 63.8 & 51.5 & 63.5 & 48.0 & 64.6 & 50.2 & 65.6 & 51.3 & 66.7 & 53.0 & 66.8 & 55.6 & 65.2 & 50.8 \\
\hline $\min (\%)$ & 59.7 & 47.5 & 57.0 & 41.2 & 57.9 & 42.5 & 59.1 & 43.8 & 62.9 & 48.5 & 62.8 & 47.3 & 58.3 & 43.1 \\
\hline avg (\%) & 61.8 & 49.6 & 60.2 & 44.5 & 61.2 & 46.2 & 62.6 & 47.5 & 65.1 & 50.8 & 65.2 & 51.8 & 61.6 & 46.6 \\
\hline stdev & 1.4 & 1.3 & 2.3 & 2.2 & 2.3 & 2.4 & 2.1 & 2.2 & 1.1 & 1.2 & 1.5 & 3.2 & 2.3 & 2.4 \\
\hline combined size (tests) & \multicolumn{2}{|c|}{615} & \multicolumn{2}{|c|}{552} & \multicolumn{2}{|c|}{1294} & \multicolumn{2}{|c|}{4002} & \multicolumn{2}{|c|}{10241} & \multicolumn{2}{|c|}{7967} & \multicolumn{2}{|c|}{3627} \\
\hline combined coverage $(\%)$ & 65.2 & 53.1 & 65.1 & 51.6 & 66.0 & 54.1 & 66.8 & 55.0 & 67.4 & 55.8 & 67.6 & 57.4 & 66.8 & 54.9 \\
\hline \multicolumn{15}{|c|}{ shortest yield embedding } \\
\hline algorithm (tests) & \multicolumn{2}{|c|}{ pll (62.0) } & \multicolumn{2}{|c|}{ rule $(48.0)$} & \multicolumn{2}{|c|}{$\operatorname{cdrc}(81.7)$} & \multicolumn{2}{|c|}{$\operatorname{step}_{3}(183.6)$} & \multicolumn{2}{|c|}{ step $_{4}(435.2)$} & \multicolumn{2}{|c|}{ deriv (439.8) } & \multicolumn{2}{|c|}{$\mathrm{bfs}_{2}(153.0)$} \\
\hline coverage type & line & branch & line & branch & line & branch & line & branch & line & branch & line & branch & line & branch \\
\hline $\max (\%)$ & 63.6 & 52.3 & 63.2 & 49.9 & 64.3 & 51.4 & 65.4 & 52.5 & 66.6 & 54.2 & 66.6 & 55.6 & 64.6 & 51.8 \\
\hline $\min (\%)$ & 61.9 & 49.7 & 61.4 & 46.8 & 62.3 & 48.2 & 63.9 & 49.6 & 65.3 & 52.0 & 65.6 & 54.2 & 62.6 & 48.5 \\
\hline $\operatorname{avg}(\%)$ & 63.0 & 51.2 & 62.5 & 48.5 & 63.5 & 50.2 & 64.7 & 51.4 & 66.0 & 53.3 & 66.2 & 55.1 & 63.8 & 50.5 \\
\hline stdev & 0.5 & 0.7 & 0.5 & 0.8 & 0.5 & 0.8 & 0.5 & 0.8 & 0.3 & 0.6 & 0.2 & 0.3 & 0.5 & 0.8 \\
\hline combined size (tests) & \multicolumn{2}{|c|}{211} & \multicolumn{2}{|c|}{198} & \multicolumn{2}{|c|}{520} & \multicolumn{2}{|c|}{1923} & \multicolumn{2}{|c|}{4953} & \multicolumn{2}{|c|}{4941} & \multicolumn{2}{|c|}{1553} \\
\hline combined coverage (\%) & 64.9 & 54.3 & 64.9 & 53.8 & 65.5 & 54.7 & 66.3 & 55.5 & 67.3 & 57.0 & 67.4 & 57.6 & 65.7 & 54.9 \\
\hline
\end{tabular}

Table 1. AMPL code coverage for different embeddings and coverage criteria. Coverage is averaged over all 61 student compilers. The minimum and maximum coverage percentages in each row are shown in italics and bold, respectively. The average sizes of the test suites for the different criteria are shown in parentheses next to the respective names. 


\begin{tabular}{|c|c|c|c|c|c|c|c|c|c|c|c|c|}
\hline \multicolumn{13}{|c|}{ shallowest embedding } \\
\hline algorithm (tests) & \multicolumn{2}{|c|}{ pll (4835.9) } & \multicolumn{2}{|c|}{ rule $(366.2)$} & \multicolumn{2}{|c|}{$\operatorname{cdrc}(1681.2)$} & \multicolumn{2}{|c|}{ step $_{3}(31330.6)$} & \multicolumn{2}{|c|}{ deriv (2961.2) } & \multicolumn{2}{|c|}{$\mathrm{bfs}_{2}(57173.6)$} \\
\hline coverage type & line & branch & line & branch & line & branch & line & branch & line & branch & line & branch \\
\hline $\max (\%)$ & 26.2 & 25.5 & 26.2 & 25.5 & 26.9 & 26.4 & 27.4 & 27.0 & 26.9 & 26.4 & 27.7 & 27.1 \\
\hline $\min (\%)$ & 22.5 & 22.1 & 22.1 & 21.7 & 23.8 & 23.4 & 25.1 & 24.7 & 24.5 & 24.1 & 24.6 & 24.0 \\
\hline $\operatorname{avg}(\%)$ & 24.4 & 23.7 & 24.0 & 23.4 & 25.3 & 24.8 & 26.5 & 26.0 & 25.9 & 25.3 & 26.1 & 25.4 \\
\hline stdev & 0.9 & 0.9 & 1.0 & 1.0 & 0.7 & 0.6 & 0.5 & 0.5 & 0.5 & 1.1 & 0.7 & 0.7 \\
\hline combined size (tests) & \multicolumn{2}{|c|}{140592} & \multicolumn{2}{|c|}{12329} & \multicolumn{2}{|c|}{72091} & \multicolumn{2}{|c|}{1683000} & \multicolumn{2}{|c|}{124401} & \multicolumn{2}{|c|}{3374922} \\
\hline combined coverage (\%) & 26.7 & 26.2 & 26.5 & 25.9 & 27.3 & 26.8 & 27.7 & 27.5 & 27.3 & 26.8 & 28.1 & 27.6 \\
\hline \multicolumn{13}{|c|}{ shortest yield embedding } \\
\hline algorithm (tests) & \multicolumn{2}{|c|}{ pll (4185.0) } & \multicolumn{2}{|c|}{ rule $(371.0)$} & \multicolumn{2}{|c|}{$\operatorname{cdrc}(1681.0)$} & \multicolumn{2}{|c|}{ step $_{3}(30896.8)$} & \multicolumn{2}{|c|}{ deriv (2992.4) } & \multicolumn{2}{|c|}{$\mathrm{bfs}_{2}(56877.0)$} \\
\hline coverage type & line & branch & line & branch & line & branch & line & branch & line & branch & line & branch \\
\hline $\max (\%)$ & 26.0 & 25.4 & 25.3 & 24.7 & 25.8 & 25.3 & 27.6 & 27.2 & 26.9 & 25.4 & 25.9 & 25.4 \\
\hline $\min (\%)$ & 23.7 & 23.3 & 23.3 & 22.8 & 24.0 & 23.7 & 24.6 & 24.5 & 24.4 & 24.9 & 25.5 & 24.9 \\
\hline $\operatorname{avg}(\%)$ & 24.4 & 23.9 & 24.0 & 23.5 & 25.1 & 24.6 & 26.7 & 26.4 & 26.0 & 25.2 & 25.8 & 25.2 \\
\hline stdev & 0.7 & 0.6 & 0.6 & 0.5 & 0.5 & 0.4 & 0.8 & 0.7 & 0.7 & 0.1 & 0.1 & 0.1 \\
\hline combined size (tests) & \multicolumn{2}{|c|}{13373} & \multicolumn{2}{|c|}{$\frac{1}{2381}$} & \multicolumn{2}{|c|}{30900} & \multicolumn{2}{|c|}{894470} & \multicolumn{2}{|c|}{56691} & \multicolumn{2}{|c|}{1746918.0} \\
\hline combined coverage (\%) & 26.3 & 25.7 & 25.5 & 24.9 & 26.0 & 25.6 & 27.8 & 27.5 & 27.4 & 26.9 & 27.1 & 26.7 \\
\hline
\end{tabular}

Table 2. SQLite code coverage for different embeddings and coverage criteria. Coverage is obtained over the SQLite executable (v3.36.0). See Table 1 for further explanations.

\section{References}

[1] Alfred V. Aho, Monica S. Lam, Ravi Sethi, and Jeffrey D. Ullman. 2007. Compilers: Principles, Techniques, \& Tools (2nd ed.). Addison-Wesley.

[2] Bernd Fischer, Ralf Lämmel, and Vadim Zaytsev. 2011. Comparison of Context-Free Grammars Based on Parsing Generated Test Data. In Software Language Engineering - 4th International Conference, SLE 2011, Braga, Portugal, July 3-4, 2011, Revised Selected Papers (Lecture Notes in Computer Science, Vol. 6940), Anthony M. Sloane and Uwe Aßmann (Eds.). Springer, 324-343. https://doi.org/10.1007/978-3-642-288302_18

[3] Nikolas Havrikov and Andreas Zeller. 2019. Systematically Covering Input Structure. In 34th IEEE/ACM International Conference on Automated Software Engineering, ASE 2019, San Diego, CA, USA, November 11-15, 2019. IEEE, 189-199. https://doi.org/10.1109/ASE.2019.00027

[4] Ralf Lämmel. 2001. Grammar Testing. In Fundamental Approaches to Software Engineering, 4th International Conference, FASE 2001 Held as Part of the foint European Conferences on Theory and Practice of Software, ETAPS 2001 Genova, Italy, April 2-6, 2001, Proceedings (Lecture Notes in Computer Science, Vol. 2029), Heinrich Hußmann (Ed.) Springer, 201-216. https://doi.org/10.1007/3-540-45314-8_15

[5] Brian A. Malloy and James F. Power. 2001. An Interpretation of Purdom's Algorithm for Automatic Generation of Test Cases. In 1st ACIS Annual International Conference on Computer and Information Science. http://eprints.maynoothuniversity.ie/6434/

[6] Paul Purdom. 1972. A Sentence Generator for Testing Parsers. BIT Numerical Mathematics 12 (Sept. 1972), 366-375. Issue 3. https://doi. org/10.1007/BF01932308

[7] Moeketsi Raselimo and Bernd Fischer. 2019. Spectrum-based fault localization for context-free grammars. In Proceedings of the 12th ACM
SIGPLAN International Conference on Software Language Engineering, SLE 2019, Athens, Greece, October 20-22, 2019, Oscar Nierstrasz, Jeff Gray, and Bruno C. d. S. Oliveira (Eds.). ACM, 15-28. https://doi.org/ 10.1145/3357766.3359538

[8] Christoff Rossouw and Bernd Fischer. 2020. Test case generation from context-free grammars using generalized traversal of LR-automata. In Proceedings of the 13th ACM SIGPLAN International Conference on Software Language Engineering, SLE 2020, Virtual Event, USA, November 16-17, 2020, Ralf Lämmel, Laurence Tratt, and Juan de Lara (Eds.). ACM, 133-139. https://doi.org/10.1145/3426425.3426938

[9] Prashast Srivastava and Mathias Payer. 2021. Gramatron: Effective Grammar-Aware Fuzzing. In Proceedings of the 30th ACM SIGSOFT International Symposium on Software Testing and Analysis (Virtual, Denmark) (ISSTA 2021). Association for Computing Machinery, New York, NY, USA, 244-256. https://doi.org/10.1145/3460319.3464814

[10] Phillip van Heerden, Moeketsi Raselimo, Konstantinos Sagonas, and Bernd Fischer. 2020. Grammar-based testing for little languages: an experience report with student compilers. In Proceedings of the 13th ACM SIGPLAN International Conference on Software Language Engineering, SLE 2020, Virtual Event, USA, November 16-17, 2020, Ralf Lämmel, Laurence Tratt, and Juan de Lara (Eds.). ACM, 253-269. https://doi.org/10.1145/3426425.3426946

[11] Sergey V. Zelenov and Sophia A. Zelenova. 2005. Automated Generation of Positive and Negative Tests for Parsers. In Formal Approaches to Software Testing, 5th International Workshop, FATES 2005, Edinburgh, UK, July 11, 2005, Revised Selected Papers (Lecture Notes in Computer Science, Vol. 3997), Wolfgang Grieskamp and Carsten Weise (Eds.). Springer, 187-202. https://doi.org/10.1007/11759744_13 